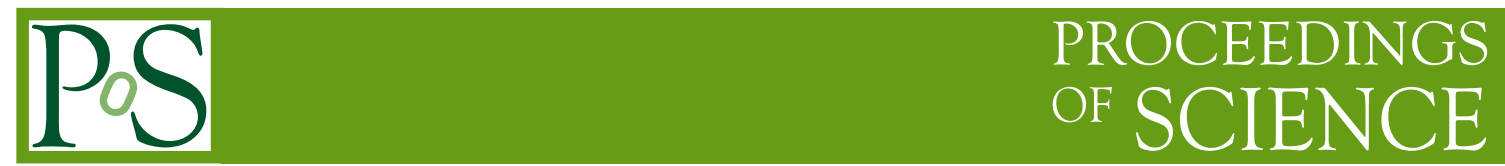

\title{
Alignment results of LHC tracking detectors
}

\author{
Ernesto Migliore* \\ Dipartimento di Fisica Sperimentale, Università di Torino e INFN Sezione di Torino \\ E-mail: miglioredto.infn.it
}

First alignment results of LHC experiments tracking detectors, based on the cosmic data taken during commissioning runs with the detectors in their final positions, are presented. The unprecedented complexity of these detectors, made of several thousands of silicon modules built in different technologies, is a challenge for alignment. Indeed, for an optimal performance, the position and orientation of the modules need to be determined with a precision of few micrometers.

VERTEX 2009 (18th workshop) - VERTEX 2009

September 13 - 182009

Veluwe, the Netherlands

*Speaker. 


\section{Introduction}

All four experiments at the Large Hadron Collider (LHC) feature complex tracking systems based on several thousands mechanically independent sensitive devices (modules), assembled into larger structures (sub-detectors). Modules are built in different detection technologies, and provide either one-dimensional measurements (e.g. single-sided microstrips) or two-dimensional measurements (e.g. pixels, double-sided microstrips, double-sided modules composed of single-sided microstrip sensors precisely mounted back-to-back, and silicon drift detectors). All these devices have a typical intrinsic precision of few tens of micrometers.

As modules are usually in positions different from their design requirement, alignment is required in order to properly reconstruct charged particles trajectories. In the case of LHC experiments, the goal of the alignment is to keep the relative contribution of the misalignment to the tracking resolution below 20\% [1]. To reach this goal, the position of the sensitive elements has be known with an uncertainty of few micrometers and their orientations with few microradians ${ }^{1}$.

Alignment of the tracking systems is achieved by two means: First, using the information collected during the different steps of the assembly (surveys) and second, by determining in-situ the position $\mathbf{p}_{m}$ of the modules using tracks, as misalignment affects the track-to-hit residuals $\mathbf{r}_{i}$. The determination of the exact position of the modules is achieved by minimizing an objective function:

$$
\chi^{2}\left(\mathbf{p}_{m}, \mathbf{q}_{\text {trks }}\right)=\sum_{\text {residuals }} \mathbf{r}_{i}^{T} \mathbf{V}_{i} \mathbf{r}_{i}
$$

built using the residuals $\mathbf{r}_{i}$ and the covariance matrix $\mathbf{V}_{i}$ of the measurements. The objective function depends also on the tracks parameters $\mathbf{q}_{t r k s}$, though these parameters are usually not explicitly determined in the alignment procedure. The optimization problem is solved assuming that the objective function can be linearized in terms of the alignment corrections $\delta \mathbf{p}_{m}=\mathbf{p}_{m}-\mathbf{p}_{m 0}$, with $\mathbf{p}_{m 0}$ being reasonable starting values for the positions of the modules. Finally the system of linear equations to be solved becomes:

$$
\delta \mathbf{p}_{m}=-\left(\left.\frac{d^{2} \chi^{2}}{d \mathbf{p}_{m}^{2}}\right|_{\mathbf{p}_{\mathbf{m} \mathbf{0}}}\right)^{-1} \frac{d \chi^{2}\left(\mathbf{p}_{m 0}\right)}{d \mathbf{p}_{m}}
$$

In case of $N$ modules with six degrees of freedom each to be determined, $\left.\frac{d^{2} \chi^{2}}{d \mathbf{p}_{m}^{2}}\right|_{\mathbf{p}_{\mathbf{m} 0}}$ is a $6 N \times 6 N$ matrix that has to be inverted. The two most common approaches for solving the problem are:

- the global method [2], where the $6 N \times 6 N$ matrix is inverted, thus accounting for the correlations between the modules. This is usually feasible by adopting an approximation of the track model used in the reconstruction (typically a Kalman filter).

- the local method, where $N$ matrices $6 \times 6$, are separately inverted thus neglecting the correlations between different modules. In this method the same track model of the reconstruction is used, but correlations between distant modules are achieved only through several iterations.

\footnotetext{
${ }^{1}$ In the LHC experiments, track parameters are usually expressed in right-handed coordinate systems, global coordinates, with the origin at the nominal collision point and with the $z$-axis directed along the beamline. The azimuthal angle $(\phi)$ is measured from the positive $x$-axis in the $x-y$ plane, whereas the radius $(r)$ denotes the distance from the $z$-axis. Local coordinate systems, defined for each module, are used instead for the reconstruction of the position of the hits. The precisely measured coordinate is usually defined as $x_{\text {local }}$.
} 
Alignment procedures were extensively tested by the four experiments using the data collected in the commissioning runs of 2008, mainly cosmic ray tracks, and were validated on the data themselves at two different levels:

- Low level, by checking the effective improvement of the post-alignment unbiased residuals, that is residuals where the hit under inspection was excluded from the track fit;

- High level, by both comparing segments of split cosmic ray tracks (see below) and analysing the residuals in overlapping regions of the detector.

The comparison of track segments exploits the long cosmic ray tracks crossing the entire volume of the detector. These tracks are split into two halves, usually at the point of closest approach to the nominal beamline. Each leg is refitted separately, and finally the five track parameters of each leg, computed at the common perigee, are compared. To better reproduce the topology of the tracks expected in collisions, tracks are usually required to cross the volume of the pixel detector. The analysis of the overlaps instead benefits of the small errors due to track extrapolation and to the presence of a small amount of material between the two measurements, thus being sensitive only to the misalignment between adjacent modules.

\section{Alignment of ALICE, ATLAS and CMS}

We start with the description of the analyses performed in ALICE, ATLAS and CMS, which have similar configurations of their tracking detectors. Data used were cosmic ray tracks, collected either with the solenoids off or on. In the pixels, the sub-detector with the smallest geometric acceptance for cosmic ray, the total number of tracks collected in 2008 was between 100000 and 200000 per experiment.

\subsection{ALICE}

The Inner Tracking System (ITS) of ALICE is composed of three different sub-detectors: two silicon pixel inner layers (240 modules), two silicon drift detectors intermediate layers (260 modules), and two silicon double-sided microstrip outer layers (1698 modules).

Alignment of ITS was performed in three steps using a global method on the data collected without magnetic field [3]. First, an internal alignment of the pixels was done followed by the alignment of the pixels with respect to strips, assuming for the positions of the strip modules those measured in the survey ( $5 \mu \mathrm{m}$ and $15 \mu \mathrm{m}$ accuracy for the modules on the ladders, and for the ladders on the structures, respectively). Finally the silicon drift detectors, which require in addition the calibration of the drift velocity and of the $t_{0}$, were aligned with respect pixels and strips. After pixels internal alignment, the standard deviation of a Gaussian fit of the track residuals distribution was $\sigma(r \phi)=26 \mu \mathrm{m}$, to be compared to $\sigma(r \phi)=17 \mu \mathrm{m}$ expected for a perfectly aligned detector.

In ALICE, the comparison of track segments was done with tracks made of at least four hits, all collected either in the pixels or in the strips. Track parameters were compared at the midplane $y=0$. Two different kinds of splitting were investigated: top versus bottom layers or inner versus outer layers. In both cases the resolution on the mismatch of the track parameters could be related through the geometry to the resolution on the local coordinate $x_{\text {local }}$ (Figure 1): 

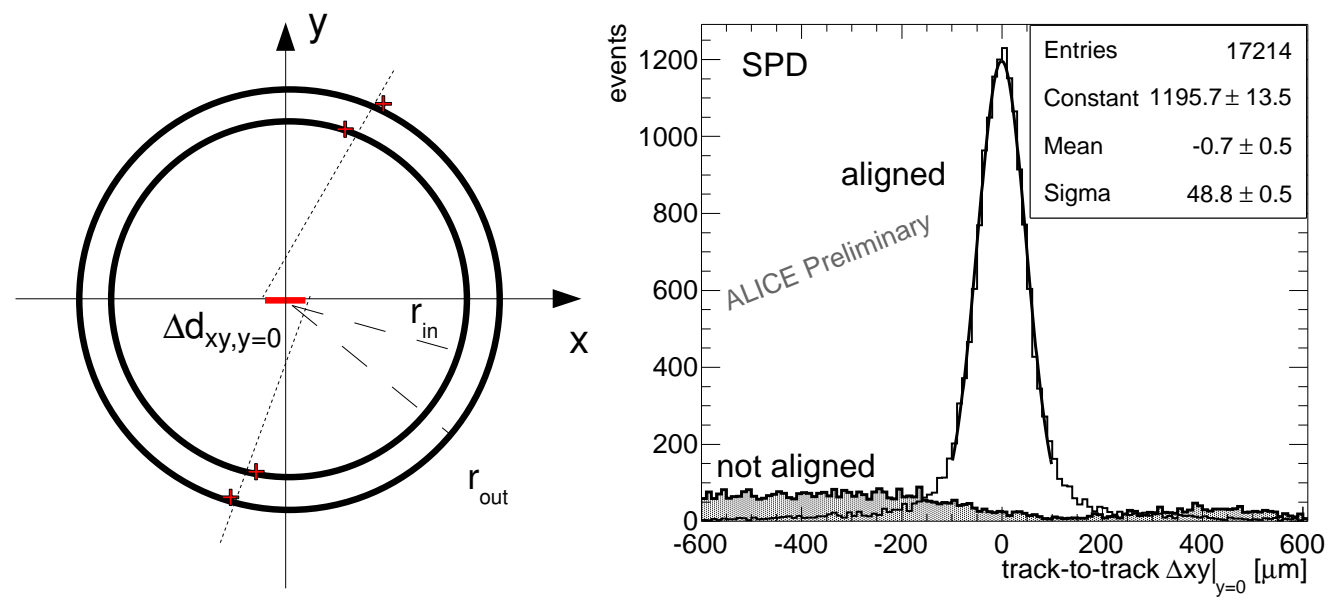

Figure 1: Definition of the mismatch $\Delta d_{x y}$ of the transverse impact parameters between the top and bottom segments of the cosmic ray track evaluated at the midplane $y=0$ (left) and its distribution measured by the pixels of the ALICE ITS (right).

$$
\sigma^{2}\left(\Delta d_{x y}\right)=2 \frac{r_{\text {out }}^{2}+r_{\text {in }}^{2}}{\left(r_{\text {out }}-r_{\text {in }}\right)^{2}} \sigma^{2}\left(x_{\text {local }}\right),
$$

in case of top-bottom layers splitting and

$$
\sigma^{2}(\Delta \phi)=\frac{1}{2}\left(\frac{1}{r_{\text {out }}^{2}}+\frac{1}{r_{\text {in }}^{2}}\right) \sigma^{2}\left(x_{\text {local }}\right),
$$

in case of inner-outer layers splitting. The standard deviation of a Gaussian fit to the distribution of transverse impact parameters mismatch $\Delta d_{x y}$ gave a resolution $\sigma\left(x_{\text {local }}\right)=14 \mu \mathrm{m}$, to be compared to $\sigma\left(x_{\text {local }}\right)=11 \mu \mathrm{m}$ expected for a perfectly aligned detector.

This value was confirmed by the analysis of overlaps, based on the track residuals computed for a second hit in a layer ("extra-cluster") which was used neither in the reconstruction of the track nor in the alignment. The Gaussian fit gave a resolution in the measurement coordinate $\sigma\left(x_{\text {local }}\right)=14 \mu \mathrm{m}$ in the pixels and $\sigma\left(x_{\text {local }}\right)=19 \mu \mathrm{m}$ in the strips, indicating a remaining misalignment less than $5 \mu \mathrm{m}$. Remarkably, the result for the strips was obtained using only the position of the modules as measured in the survey.

\subsection{ATLAS}

The ATLAS Inner Detector (ID) is composed by a pixel detector, with three barrel layers and three disks per each end-cap region, and a strip detector (SCT), made of four layers of double-sided modules in the barrel, and nine disks in each of the two endcaps. In total there are 1788 pixel and 4088 strip modules. The system is surrounded by layers of straw tubes forming the Transition Radiation Tracker (TRT) which will not be discussed here.

The ID barrel alignment was performed in three steps, from larger structures down to the module level. Survey data were used as starting point. Both alignments with the local and with the 

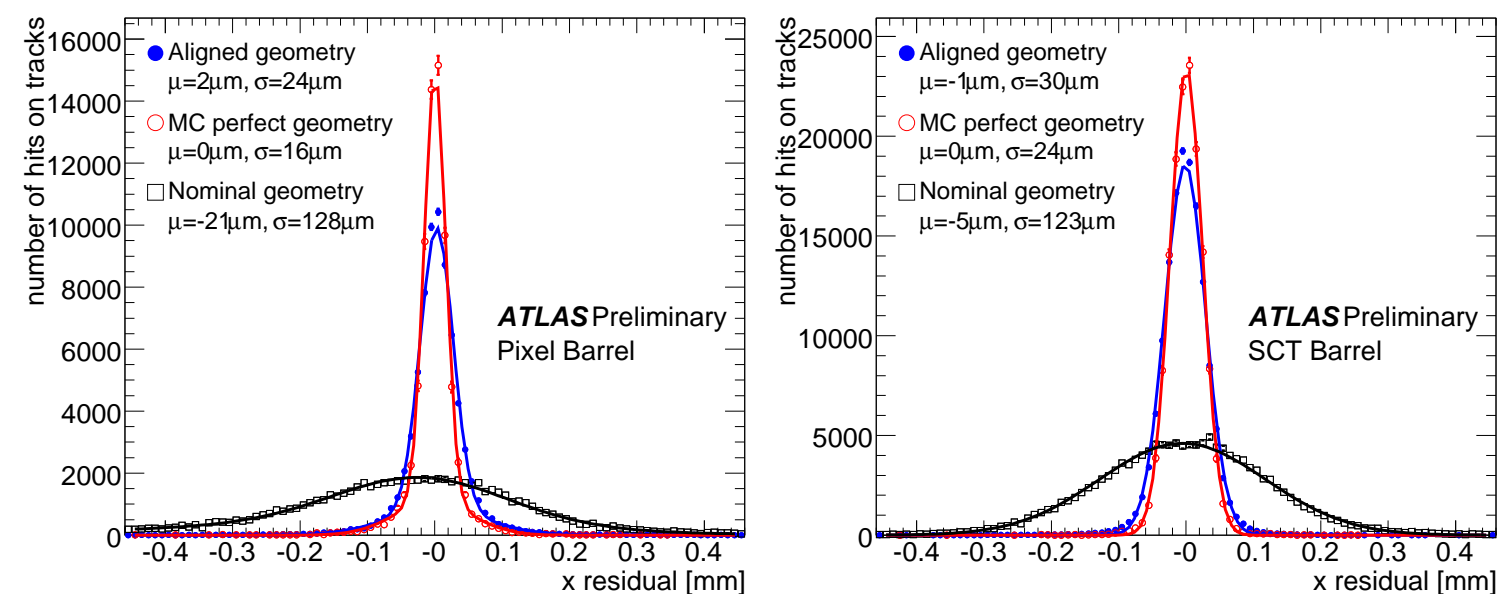

Figure 2: Distributions of the unbiased track residuals in ATLAS barrel for pixel and SCT.

global method were performed with the best results being obtained by the latter [何. Performance of the low level validation were evaluated from the distributions of the track residuals fitted by a sum of two Gaussians (Figure 2). The standard deviations of the innermost Gaussian were $\sigma\left(x_{\text {local }}\right)=24$ $\mu \mathrm{m}$ for the pixel and $\sigma\left(x_{\text {local }}\right)=30 \mu \mathrm{m}$ for the SCT. The comparison with a detailed detector simulation which assumes a perfectly aligned detector indicates a remaining module-to-module random misalignment of about $20 \mu \mathrm{m}$.

Also in ATLAS the comparison of track segments was performed at midplane $y=0$. Only tracks with transverse momentum $p_{T}>2 \mathrm{GeV} / c$ and with at least three pixel hits, with one at least in the barrel pixels, were used. Since tails were low, the mismatch of the longitudinal $d_{x y}$ and transverse $d_{z}$ components of the impact parameter could be fitted by a single Gaussian with standard deviations $\sigma\left(\Delta d_{x y}\right)=49 \mu \mathrm{m}$ and $\sigma\left(\Delta d_{z}\right)=166 \mu \mathrm{m}$, respectively.

\subsection{CMS}

The CMS inner tracking system (Tracker) consists of a silicon pixel detector surrounded by a complex of silicon strip detectors. Pixel detector is composed of three barrel layers (BPIX) and two endcaps (FPIX) with two disks each. The strip detector is made of four sub-detectors: the Tracker Inner and Outer Barrel (TIB and TOB), with four and six layers respectively, the Tracker Inner Disks (TID) and the Tracker Endcaps (TEC) made of three and nine disks respectively. In total there are 1440 pixel and 15148 strip modules.

Both a local and a global method were used for the alignment of the Tracker, but the best performance were obtained with a combined method where the local method was run on the geometry obtained via the global one [5]. Because of the large number of degrees of freedom to be determined, about 100000 , the alignment performance was asserted by looking at the median of the distribution of the post-alignment track residuals for all the modules. Distributions of the median of the residuals in BPIX and TOB are shown in Figure 3. Here the figure of merit is the RMS of the distributions, as stochastic effects, like multiple Coulomb scattering or the intrinsic resolution on the hit, which dominates the width of the distribution of post-alignment residuals, cancel out. 

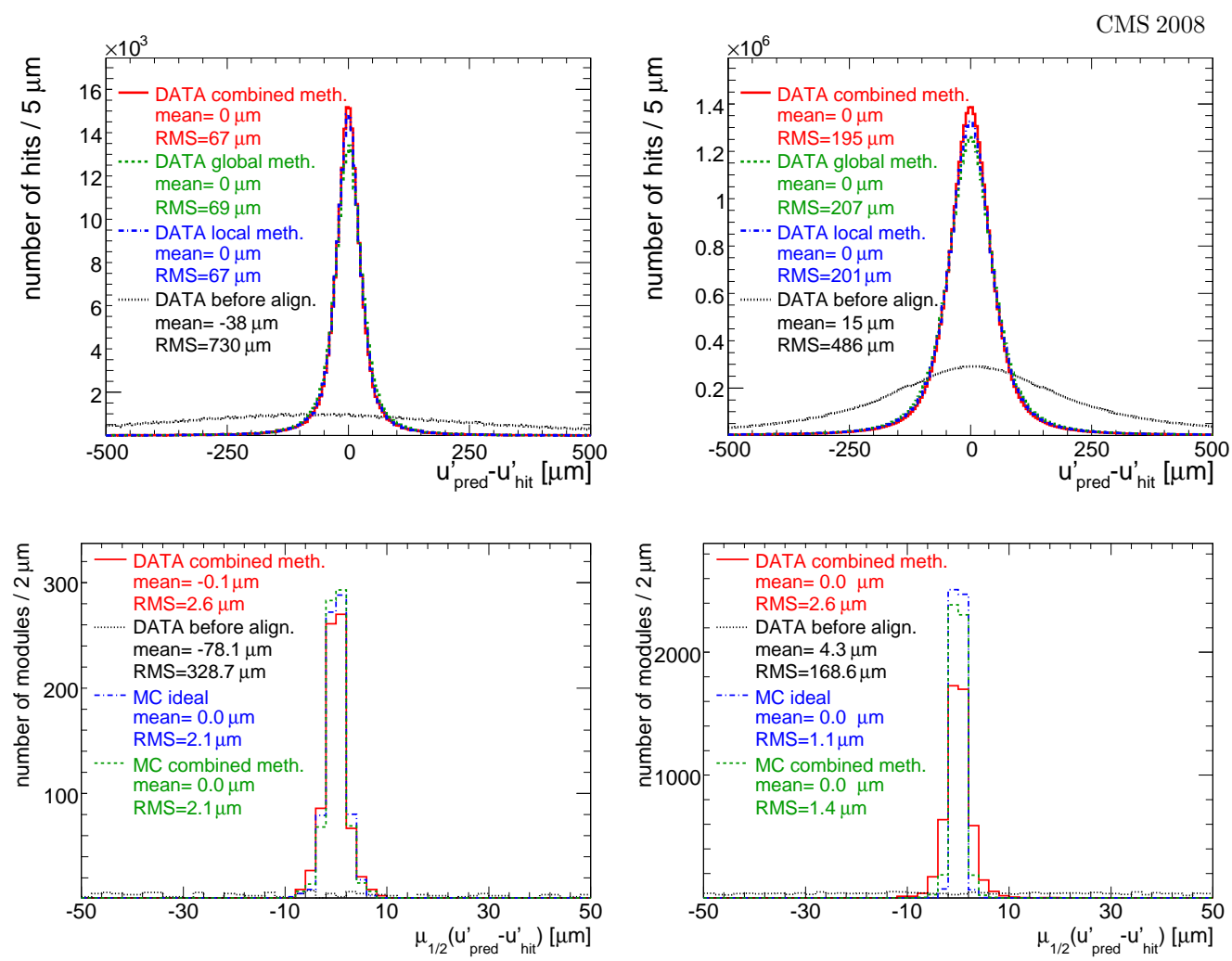

Figure 3: Distribution of the unbiased track residuals in CMS, shown for BPIX (top left) and TOB (top right), and compared with the distribution of the median of the residuals $\left(\mu_{1 / 2}\right)$ in the same sub-detectors, BPIX (bottom left) and TOB (bottom right). The $u^{\prime}$ coordinate corresponds to $x_{\text {local }}$ but with a direction defined to be always positive in $\phi$. In the distributions of the medians only modules with more than 30 hits are shown. Despite the difference in the spread of the distributions of the residuals of BPIX and TOB even after the alignment, the RMS of the post-alignment distributions of $\mu_{1 / 2}$ are similar thus indicating similar performance of the alignment reached in the two sub-detectors.

In CMS the comparison of the track segments was done by splitting the tracks at the point of closest approach to the nominal beamline, and then by selecting only tracks with transverse momentum $p_{T}>4 \mathrm{GeV} / c$ and with at least three pixel hits in each segment. The RMS of differences between upper and lower track segment parameters measured at the common perigee and scaled by $1 / \sqrt{2}$, were found to be $0.000864 c / \mathrm{GeV}(0.000836 \mathrm{c} / \mathrm{GeV})$ for the curvature $1 / p_{T}, 29 \mu \mathrm{m}$ $(29 \mu \mathrm{m})$ for the transverse impact parameter $d_{x y}$, and $44 \mu \mathrm{m}(41 \mu \mathrm{m})$ for the longitudinal impact parameter $d_{z}$, where the values in parentheses are those expected from a simulation with ideal detector geometry.

Finally a validation of the alignment was made by looking at the double difference between the predicted and the measured positions of the two hits in the overlap. Non zero means of the Gaussian fits to these distributions are an indication that a relative shift between adjacent modules was not corrected by the alignment procedure. The RMS of the distributions of the shifts for $x_{\text {local }}$ scaled by $1 / \sqrt{2}$ are in the range 5-7 $\mu \mathrm{m}$, slightly larger than the values found for the distribution of the median of the residuals, possibly indicating yet unquantified systematics effects, like for 

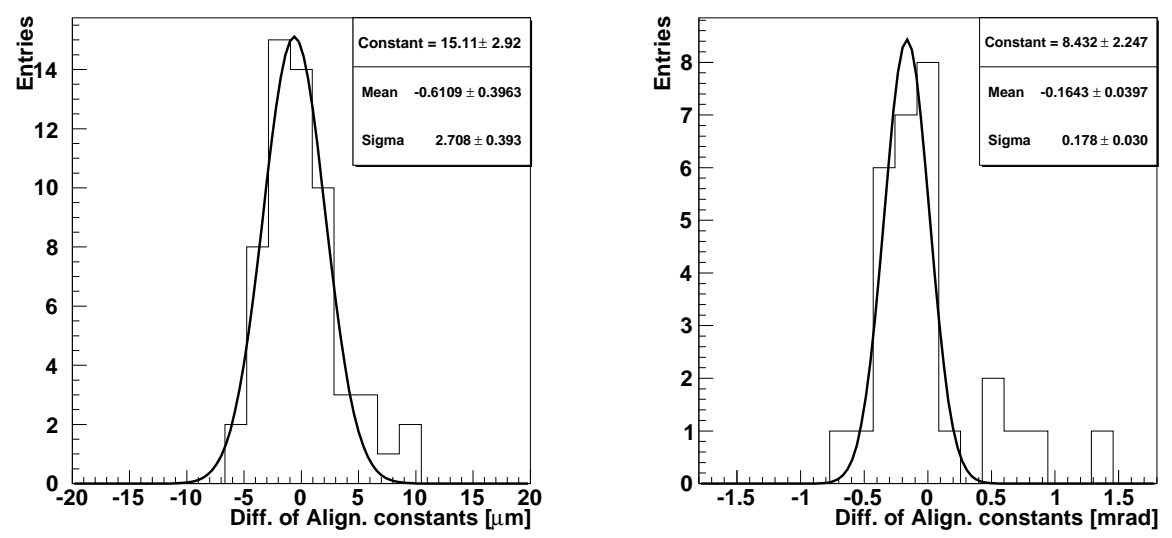

Figure 4: Distributions of the differences between the alignment constants of the LHCb VELO evaluated by the global method [G] using two different samples of data collected in 2008: $x$ and $y$ translations (left), and rotation around the $z$-axis $\phi_{z}$ (right).

example the aplanar distortion of modules.

\section{Alignment of LHCb VELO}

The LHCb VErtex LOcator (VELO) consists in two halves of 21 double-sided silicon strips modules. Because the modules are small and placed vertically, cosmic ray tracks are scarcely useful for alignment. Alignment of VELO in 2008 was instead performed with about 2000 tracks produced on a beam-dump (TED) located about $350 \mathrm{~m}$ downstream of LHCb. As particles from TED cross $\mathrm{LHCb}$ in a direction opposed to the one expected for collisions, a standalone reconstruction of tracks, based only on the VELO hits, was used.

The VELO alignment was performed at the module level, using two different implementations: one using the global method [6], the other using LHCb track model (Kalman filter) [7]. For each module two translations, $x$ and $y$, in the plane of the module and one rotation $\phi_{z}$ around the beamline were determined. As tracks were reconstructed using only hits recorded in the VELO, use of data from survey was needed to remove global deformations (e.g. shearing $\Delta x=c_{1} z$ ).

Good agreement was found between the constants determined by the two methods, better than $5 \mu \mathrm{m}$, and between the constants obtained by the track-based methods and those from survey, better than $10 \mu \mathrm{m}$. The reproducibility of the alignment constants extracted in two different sets of data is shown in Figure $\theta$.

\section{Readiness of ATLAS and CMS for collisions}

The physics performance expected at the LHC startup, after the alignment of ATLAS and CMS tracking detectors done with cosmic rays data, is best represented by the resolution on the track parameters derived from the comparison of the track segments and shown in Figure 5. In particular the resolutions both on the momentum and on the impact parameter show the expected 

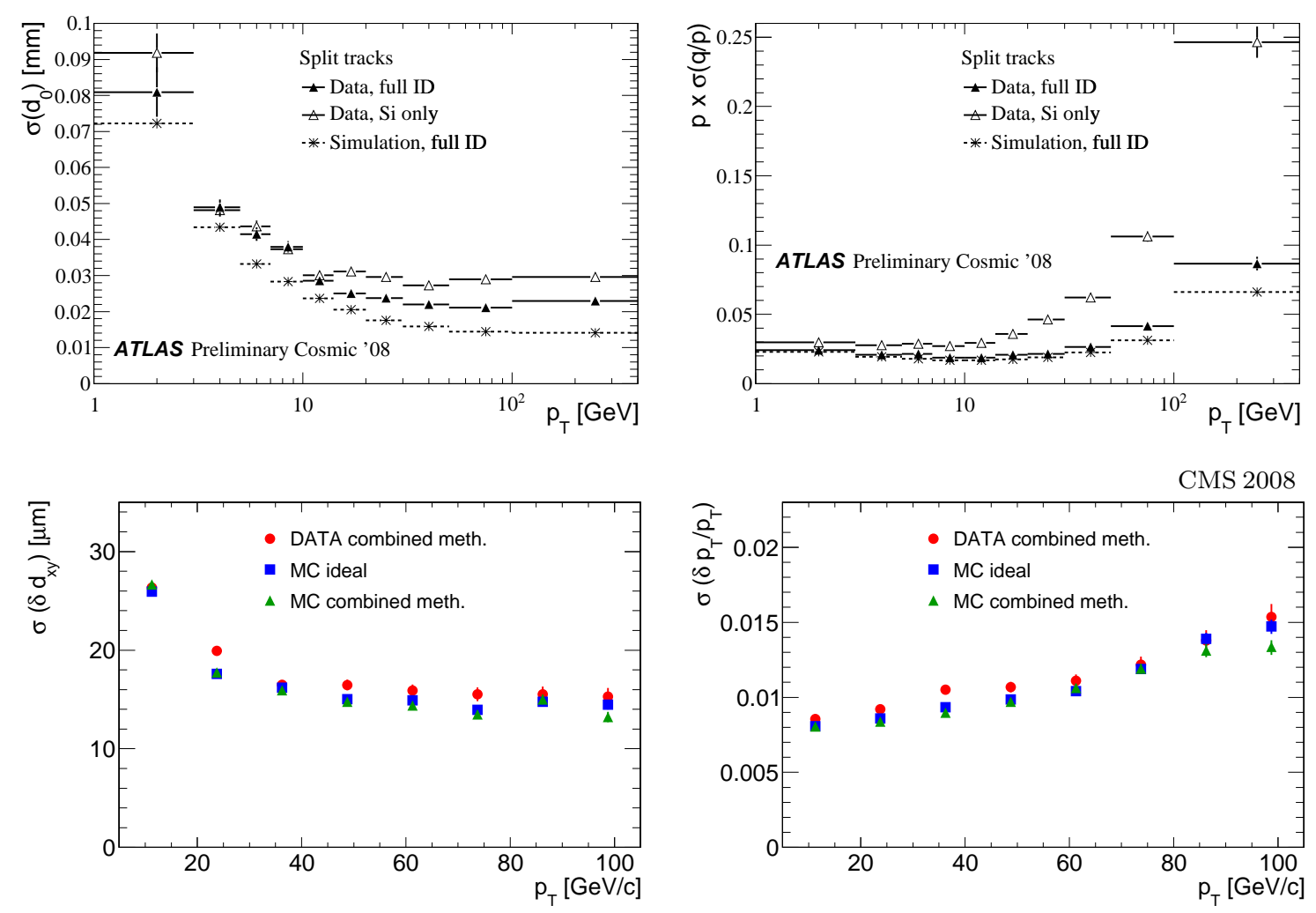

Figure 5: Dependence on $p_{T}$ of the differences between the track parameters derived from the comparison of the two halves of a split cosmic ray track and scaled by $1 / \sqrt{2}$ : ATLAS (top row) and CMS (bottom plots). Expectations for a perfectly aligned detector as determined from simulation are shown for comparison.

behavior as a function of $p_{T}$, showing the onset of the contribution of multiple Coulomb scattering at low $p_{T}$ values. Both for ATLAS and CMS the performance approach those expected on a sample of cosmic ray events reconstructed with a perfectly aligned detector.

ATLAS and CMS also have investigated possible "weak modes", that is systematics distortions which leave the track residuals unchanged but introduce systematic biases in the track reconstruction. Weak modes typically arise when a sample of tracks of only one topology is used in the alignment.

Following the approach of Ref. [8], CMS applied nine systematic distortions, in $\Delta r, \Delta \phi$ and $\Delta z$ as a function of $r, \phi$ and $z$, to the aligned geometry obtained with cosmic ray data. The systematically misaligned geometries were then used as starting point for repeating the alignment. Finally the nine geometries obtained after the new alignments were compared to the original aligned geometry to see if the distortions could be recovered by the alignment procedure. It was found that usually the $\Delta \phi$ deformations, like the layer rotation $\Delta \phi=c_{0}+c_{1} r$, are reflected in a degradation of the track $\chi^{2}$ and then can be recovered using cosmic ray tracks. The $\Delta z$ distortions instead, like the $z$-expansion $\Delta z=c_{1} z$, change only marginally the track $\chi^{2}$ thus not being recovered.

The effect of a layer rotation not yet corrected at the LHC startup was investigated in ATLAS [9]. Since a layer rotation gives opposite biases to the reconstructed $p_{T}$ of positively and negatively charged particles, a remaining distortion degrades the resolution on the mass of $Z \rightarrow \mu^{+} \mu^{-}$ 
to $2.67 \mathrm{GeV} / c^{2}$ at the startup ( $32 \%$ deterioration), being reduced to $2.42 \mathrm{GeV} / c^{2}$ after one million collected high $p_{T}$ muon tracks (20\% deterioration). This value is close to the $2.29 \mathrm{GeV} / c^{2}$ resolution expected from the remaining random module-to-module misalignment after 100 days of collisions data taking (14\% deterioration), indicating that systematic errors will become soon the dominant source of uncertainty.

\section{Acknowledgments}

The author is indebted to the colleagues A. Dainese (ALICE), S. Marti i Garcia and J. Schieck (ATLAS), S. Borghi and W. Hulsbergen (LHCb), for the help in preparing the talk and the proceedings.

\section{References}

[1] "The 3rd LHC detector alignment workshop", http://indico.cern.ch/conferenceDisplay.py?confId=50502.

[2] V. Blobel, Software alignment for tracking detectors, Nucl. Instr. Methods Phys. Res. A 566, 5, 2006.

[3] C. Bombonati et al., Alignment of the ALICE Inner Tracking System with cosmic-ray tracks, ALICE-INT-2009-035 (2009).

[4] P. Bruckman, A. Hicheur and S. Haywood, Global $\chi^{2}$ approach to the alignment of the ATLAS silicon tracking detectors, ATL-INDET-PUB-2005-002 (2005).

[5] The CMS Collaboration, S. Chatrchyan et al., Alignment of the CMS silicon tracker during commissioning with cosmic rays, arXiv:0910.2505 [physics.ins-det].

[6] S. Viret, C. Parkes and M. Gersabeck, Alignment procedure of the LHCb vertex detector, Nucl. Instr. Methods Phys. Res. A 596, 157, 2008.

[7] W. D. Hulsbergen, The global covariance matrix of tracks fitted with a Kalman filter and an application in detector alignment, Nucl. Instr. Methods Phys. Res. A 600, 471, 2009.

[8] D. N. Brown, A. V. Gritsan, Z. J. Guo, and D. A. Roberts, Local alignment of the BABAR silicon vertex tracker, Nucl. Instr. Methods Phys. Res. A 603, 467, 2009.

[9] The ATLAS Collaboration, The impact of Inner Detector misalignment on selected physics processes, ATL-PHYS-PUB-2009-080 (2009). 